\title{
Frontotemporal dementia risk variant accelerates cognitive decline in Parkinson disease
}

\section{ce}

\section{The presence} of the FTD risk variant was associated with faster cognitive decline

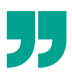

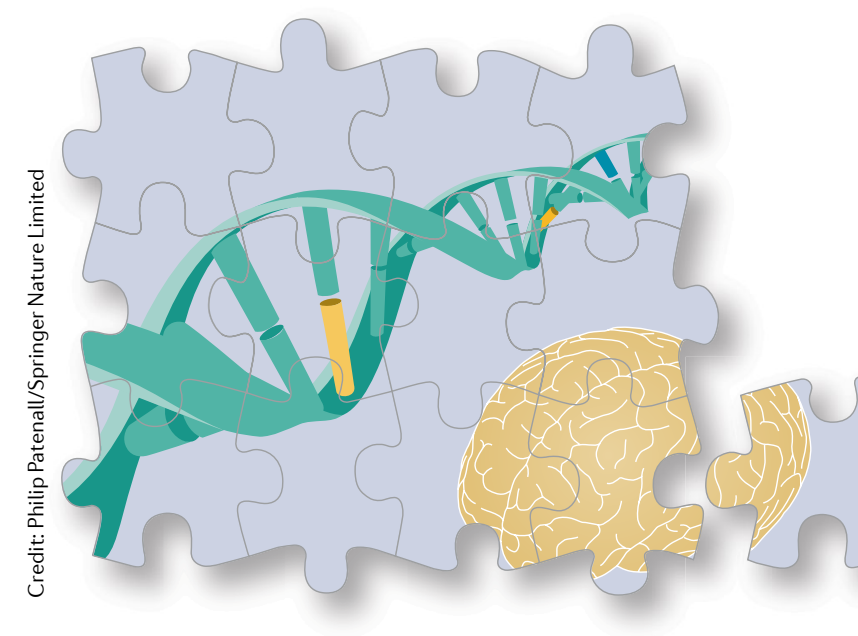

lysosomal phenotypes. "Lysosomal function is probably important not only in FTD but in many neurodegenerative diseases that have the common feature of misfolded proteins," explains Alice Chen-Plotkin, who led the new study. "We looked across multiple different neurodegenerative diseases to see if variants in TMEM106B modified the course of disease."

In the new study, Chen-Plotkin and her team measured cognitive ability with the Mini-Mental State Examination (MMSE) in a cohort of individuals already enrolled in research studies at the University of Pennsylvania, USA. The cohort included a total of 867 individuals with FTD, PD, AD or mild cognitive impairment (MCI) and 137 healthy controls. They monitored the members of this cohort for a median of 3 years (maximum 10 years) and repeated the MMSE annually during this period. The team combined this long-term cognitive assessment with genotyping for a TMEM106B variant that has previously been associated with an increased risk of FTD.

The presence of the FTD risk variant was associated with faster cognitive decline in the patients with $\mathrm{PD}$ as well as in the patients with FTD, but not in the patients with $\mathrm{AD}$ or MCI. Cognitive decline was not seen in healthy controls during the study period. In a surprise for the team, the biggest effect of TMEM106B genotype was on PD, and this effect was specific to the cognitive aspect of PD - the rate of

change in motor function was not associated with TMEM106B genotype.

Chen-Plotkin and her team then carried out a similar longitudinal study using a different cognitive test, the Mattis Dementia Rating Scale-2 (DRS-2), in which individual cognitive domains are scored. The cohort for this study was composed of 128 individuals with PD who were enrolled in studies at the University of Pennsylvania, including some individuals who took part in the initial MMSE study. The same association of TMEM106B genotype with cognitive decline was present in individuals with PD regardless of the test used, and the attention, conceptualization and memory domains were affected.

Last, the team sought to replicate their findings in a broader, international cohort, which included 371 patients with PD from 11 countries. In this cohort, they studied a different FTD risk variant of TMEM $106 \mathrm{~B}$ and found that this variant was also associated with faster rates of cognitive decline.

Whereas TDP 43 proteinopathy is a key feature of FTD, ALS and $\mathrm{AD}$, this pathology is not common in $\mathrm{PD}$, so the new results suggest that TMEM106B influences diseases via TDP43-dependent and TDP43-independent mechanisms. "Increasingly, lysosomal function (or dysfunction) has been shown to be important in the development of $\mathrm{PD}$, so this suggests that the effects of TMEM106B on the lysosomes may play a role in how PD progresses," notes Chen-Plotkin. "It also suggests that targeting TMEM106B may be therapeutically helpful not only in FTD but in PD as well."

Sarah Lemprière

ORIGINAL ARTICLE Tropea, T. F. et al. TMEM106B effect on cognition in Parkinson's disease and frontotemporal dementia. Ann. Neurol. https:// doi.org/10.1002/ana.25486 (2019) FURTHER READING Aarsland, D. et al. Cognitive decline in Parkinson disease. Nat. Rev. Neurol. 13, 217-231 (2017) 